\title{
New Technologies for Outcome Measures in Glaucoma: Review by the European Vision Institute Special Interest Focus Group
}

\author{
Ghislaine L. Traber ${ }^{a, b} \quad$ Maria della Volpe-Waizel ${ }^{a, b} \quad$ Peter Maloca $^{b}$ \\ Ursula Schmidt-Erfurth $^{c}$ Gary Rubin $^{d}$ Botond Roska ${ }^{b}$ M. Francesca Cordeiro ${ }^{d-f}$ \\ Tilman Otto $^{g}$ Richard Weleber $^{\mathrm{h}}$ Luis Andres Lesmes ${ }^{i}$ Angelo Arleo $^{\mathrm{j}}$ \\ Hendrik P.N. Scholla, b \\ ${ }^{a}$ Department of Ophthalmology, University Hospital Basel, University of Basel, Basel, Switzerland; ${ }^{b}$ Institute of

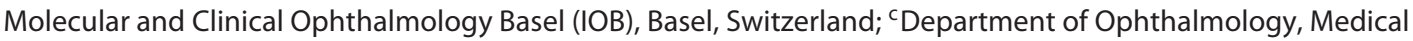 \\ University of Vienna, Vienna, Austria; ${ }^{\mathrm{d}}$ Institute of Ophthalmology, UCL University College London, London, UK; \\ eWestern Eye Hospital, Imperial College Healthcare NHS Trust, London, UK; f Imperial College Ophthalmic Research \\ Group (ICORG), Imperial College London, London, UK; ${ }^{9}$ Heidelberg Engineering GmbH, Heidelberg, Germany; ${ }^{\mathrm{h}}$ Casey \\ Eye Institute, Departments of Ophthalmology and Molecular and Medical Genetics, University of Oregon Health \& \\ Science University, Portland, OR, USA; 'Adaptive Sensory Technology, Inc., San Diego, CA, USA; jinstitut de la Vision, \\ CNRS, INSERM, Sorbonne Université, Paris, France
}

\section{Keywords}

Glaucoma · Retinal ganglion cell · Neurodegeneration · Outcome measure $\cdot$ Structure-function correlation

\begin{abstract}
Glaucoma is the leading cause of irreversible blindness worldwide, with an increasing prevalence. The complexity of the disease has been a major challenge in moving the field forward with regard to both pathophysiological insight and treatment. In this context, discussing possible outcome measures in glaucoma trials is of utmost importance and clinical relevance. A recent meeting of the European Vision Institute (EVI) special interest focus group was held on "New Technologies for Outcome Measures in Retina and Glaucoma," addressing both functional and structural outcomes, as well as translational hot topics in glaucoma and retina research. In conjunction with the published literature, this review summarizes the meeting focusing on glaucoma.
\end{abstract}

(c) 2020 S. Karger AG, Basel

\section{KARGER}

(c) 2020 S. Karger AG, Basel

E-Mail karger@karger.com

www.karger.com/ore

\section{Introduction}

Worldwide, glaucoma is the leading cause of irreversible blindness [1]. The hallmark of the disease is the degeneration of retinal ganglion cells and its axons, resulting in progressive optic neuropathy with typical changes to the optic disc $[2,3]$. Glaucomatous neurodegeneration is related to the level of intraocular pressure, albeit also independent thereof, and even does not seem to be confined to the anterior visual pathway [4]. Still, lowering intraocular pressure currently is the only proven therapeutic option $[2,5]$.

Given its age-related nature, this global epidemic is still on the rise, not only causing significant visual disability but also adding further to our health-economic burden [6]. The complexity of the disease has been a major challenge in moving the field forward, both with regard to pathophysiological insight and treatment. Considering the slow progression of the disease, realization of glau-

Hendrik P.N. Scholl, MD, MA

Institute of Molecular and Clinical Ophthalmology Basel (IOB) Mittlere Strasse 91

CH-4031 Basel (Switzerland)

E-Mail hendrik.scholl@iob.ch 
coma trials is further hampered by their duration, required sample size, and resulting costs. In this context, a discussion of possible outcome measures is highly relevant in order to possibly clear these hurdles by improving the sensitivity of surrogate endpoints.

A recent meeting of the European Vision Institute (EVI) special interest focus group was held on "New Technologies for Outcome Measures in Retina and Glaucoma," hosted by the Institute of Molecular and Clinical Ophthalmology Basel (IOB), University of Basel, Switzerland, November 8-9, 2018.

The agenda with presentations of international clinicians and scientists was selected by Hendrik Scholl and the EVI steering committee. Backed up by published literature, the presentations and discussions are summarized below, focusing on glaucoma.

\section{Presentation Summaries}

\section{Active Learning for Precision Measurement of Visual Function}

With the idea of improving the precision of vision testing, Luis Andres Lesmes and coworkers from Adaptive Sensory Technology, Inc., introduced the concept of active learning, which improves the assessment of contrast sensitivity (CS) and visual acuity (VA) through the combination of digitized displays, novel quantitative Bayesian models of visual function, and intelligent sampling algorithms that personalize testing to each patient. For each patient, the active learning algorithm evaluates an expansive space of potential test outcomes, searches a large library of potential contrast and acuity test items, and converges them into a test sequence comprising optimal queries for each patient based on their previous responses.

The CS function (CSF) is fundamental to vision science, a threshold contour that represents the boundary between what one can see in the world and what one cannot see (Fig. 1, left). Rather than estimate contrast sensitivities for individual spatial frequency conditions, the quick CSF (qCSF) directly estimates a Bayesian parameterization of the function's global shape. Using the higher pixel and contrast resolution of digital displays, as well as an intelligent algorithm for sampling stimulus size and contrast, the $\mathrm{qCSF}$ algorithm presents the patient with the size-contrast combinations that comprise an optimal assessment of the personalized shape of the patient's CSF (Fig. 1, right). The CSF shows disease-specific patterns of function loss in various ophthalmic conditions including glaucoma [7-13] and might thus serve as a valuable out-

New Technologies for Outcome Measures in Glaucoma come measure by revealing hidden signals of early vision loss and by improving the statistical power for detecting visual changes through a better signal-to-noise ratio [14].

VA testing has typically implemented two complementary but exclusive strategies: (1) static, chart-based testing that presents optotypes of fixed sizes in rows (ETDRS and Pelli-Robson charts), or (2) computerized testing that applies a staircase to change the size of single optotypes [15]. Lesmes and Dorr [16] have proposed a quantitative VA (qVA) algorithm which combines these approaches to change the size of multiple optotypes, using a row-based psychometric function of expected correct optotypes as a function of logMAR optotype size. With intelligent sampling of optotype size (true 0.02$\log$ MAR resolution), the algorithm reduces the noise in VA testing and provides fine-grained information on the threshold and range of the VA psychometric function.

The qCSF and qVA algorithms have exhibited potential to improve the statistical power for reliably detecting subtle but potentially clinically significant signals in visual function. Because the definition of a clinically meaningful change is constrained by what is clinically measurable, there is potential for novel methodology to drive the threshold of what is accepted both by clinicians and by regulatory authorities as clinically relevant.

\section{Microperimetry as a Visual Function Endpoint}

Gary Rubin's talk focused on microperimetry. There are currently three different commercially available microperimeters. The NIDEK MP-3 (NIDEK Co. Ltd, Gamagori, Japan) and the MAIA (CenterVue, Padua, Italy) combine microperimetry with nonmydriatic color fundus imaging and scanning laser ophthalmoscopy (SLO) imaging, respectively. The OptosOCT SLO (Optos PLC, Dunfermline, UK) is the first device to superimpose microperimetry data on OCT images. Eye tracking allows the projection of stimuli onto different retinal locations with imaging of the fundus in real time. Microperimetry is thus providing structure-function correlations and is particularly useful in patients with unstable or eccentric fixation. The technology has mainly been applied in retinal diseases.

Gary Rubin comments on its use in the EFFECT (Eccentric Fixation from Enhanced Clinical Training) trial on recent-onset macular disease (Rubin G. First results from the EFFECT Trial, an RCT of eccentric viewing training for patients with AMD, ARVO Annual Meeting Abstract, June 2017, Baltimore, MD, USA, May 7-11, 2017. Invest Ophthalmol Vis Sci. 2017 Jun;58:4766) [17] and in gene therapy trials on Leber's congenital amauro- 


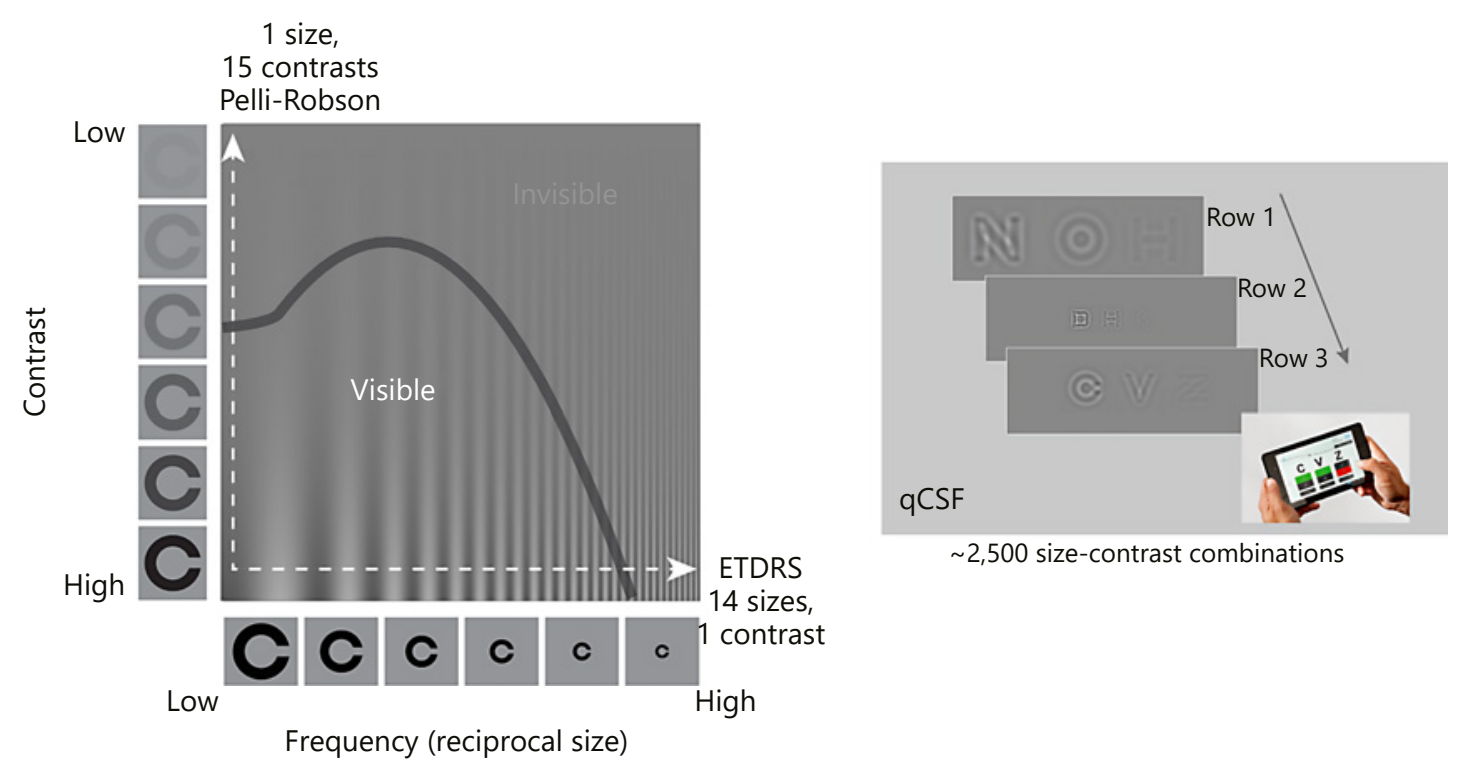

Fig. 1. Quick contrast sensitivity function (qCSF). Left: it has long been recognized that optotype size and contrast are critical attributes of clinical vision testing, with ETDRS and Pelli-Robson charts for acuity and contrast sensitivity following complementary strategies of fixing one and testing the other. This coarse, piecemeal approach neglects the broader information conveyed by the CSF, a two-dimensional threshold contour that describes how size and contrast affect the visibility of spatial patterns. Right: the qCSF

applies active learning to personalize and directly estimate the global shape of the CSF, by intelligent sampling of size-contrast test patterns that effectively trace out the boundary between visible and invisible for each patient. During testing, a technician enters the responses of patients presented with three special optotypes, with size-contrast combinations selected to provide an optimal, personalized sequence with sampling from above and below the threshold contour.

sis $[18,19]$. However, microperimetry has also been investigated in glaucoma. The published literature highlights aspects of interest including a comparison with standard automated perimetry [20-22], structure-function correlations [22-26], assessment of early glaucoma detection [27], visual field evaluation in advanced glaucoma $[28,29]$, and fixation instability in glaucoma $[30$, 31].

\section{Modeling and Analysis of the Hill of Vision of}

Full-Field Static Perimetry

On behalf of Richard Weleber, Hendrik Scholl explained the concept of visual field modeling and analysis (VFMA). The hill of vision (HOV) is a functional measure of the total light sensitivity across the retina, which VFMA visualizes via a color-coded $3 \mathrm{D}$ sensitivity surface. A single volumetric measure in decibel-steradian (dB-sr) units quantitatively estimates the HOV. As opposed to conventional visual field indices (e.g., mean sensitivity, mean defect, and square root of loss variance), VFMA not only captures the whole visual field sensitivity in one sin-

gle endpoint (dB-sr), but also has a flexible region of interest and is more robust with regard to grid changes. The latter is explained by the fact that HOV computation is achieved by interpolated dense VFMA gridding based on sparse gridding of the measured raw data. As a consequence, the HOV volume is a flexible and robust measure of quantity resulting in a stronger, more focused endpoint.

The methodology was established in 2015 [32] and since then has been used in several trials including the RPE65 gene therapy trial [33]. VFMA mainly has been adopted in trials on retinal disease, but it could without doubt be established in glaucoma as well. Standard visual field testing frequently fails to detect early arcuate glaucomatous visual field loss in the central macula just superior to fixation [24]. Since the respective retinal ganglion cells of the inferior macula are particularly vulnerable to glaucomatous damage, additional screening of this area with condensed stimulus arrangements has been suggested. However, the utility of this approach still has to be substantiated, since grids of greater spatial density in re- 


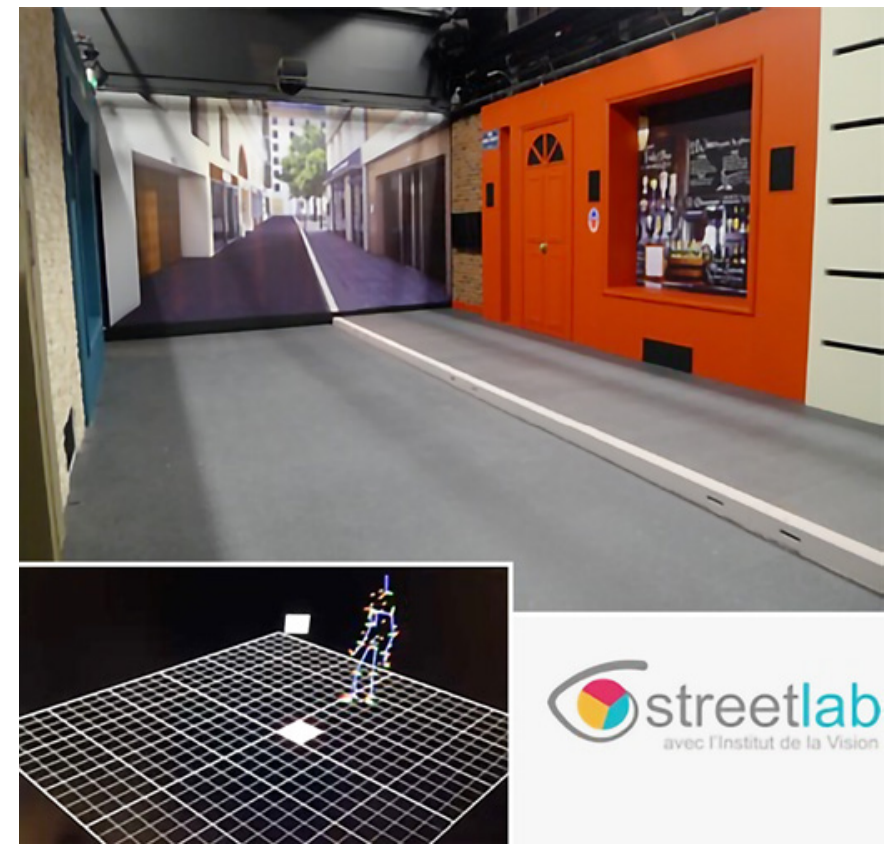

Fig. 2. Ecological, real environment for naturalistic behavioral measures. The Streetlab platform (Vision Institute; www.streetlabvision.com) was used to assess the visuospatial behavior of young and older participants. The Streetlab reproduced a $10 \times 5$ portion of a street-like environment. It ensured visual immersion through realistic relief elements (imitating doors, windows, and brick walls), and audio immersion through a $3 \mathrm{D}$ multisource sound system. The floor was covered by a black linoleum surface and there were no obstacles. Light conditions were fully controlled by varying both intensity and temperature parameters. The subject's whole body and eye movements was monitored through a set of biometric sensors: an optoelectronic Vicon motion capture system (10 infrared cameras, at a sampling frequency of $120 \mathrm{~Hz}$ ) and a wearable eye tracking system (by MocapLab, at $60 \mathrm{~Hz}$ ). Data from all sensors were synchronized and recorded in real time, allowing any kinematic recording to be replayed and analyzed offline. Adapted, with permission, from Streetlab ${ }^{\complement}$.

gions of interest do break the usefulness of the conventional visual field indices in their ability to compare to fields with standard grids.

\section{Behavioral Outcome Measures and Naturalistic Testing}

Angelo Arleo presented his group's research on the visuospatial behavioral performance of young versus elderly subjects. The presented data mainly focused on healthy ageing, and they serve as a scientific basis for the future assessment of visuospatial behavior in patients with visual or cognitive impairment. Age-related decline of visuospatial function is related to optical, neurosensory, and cognitive factors. The experimental setup is designed for cognitive aspects and analyzes how age interferes with spatial perception, spatial action, and spatial learning, which might serve as a functional marker of mobility and autonomy loss in the elderly.

Our internal representation of space is anchored in different types of visual information, which is referred to as spatial coding. In order to avoid the possible bias induced by computer screen-based tasks like lack of multisensory integration, coordinate transformation, and a limited field of view, Arleo and coworkers established an ecological experimental setup (Fig. 2) to study spatial learning and to find out what type of visual information different age groups rely on. Assessed in a symmetric $\mathrm{Y}$-shaped maze, vector field analysis of age groups showed that children and healthy elderly adults mainly navigate in an egocentric manner, which is referred to as a loss of allocentric strategies with age in the literature [34]. However, they become able to use allocentric information in an asymmetric Y-shaped maze $[35,36]$. By contrast, young adults rely on allocentric navigation under both conditions $[35,36]$. Arleo and coworkers hypothesize that this is more likely related to visual and spatial cue processing than to strategic choices (egocentric vs. allocentric). Exploring a naturalistic maze, children and elderly adults again behave similarly, and mainly depend on the geometry of the environment, while young adults mainly focus on landmarks. The latter visual behavior is corroborated by analysis of visual exploration patterns using eye tracking, which predicts spatial cue preference (geometry vs. landmarks). Extensive visual function and neuropsychological testing did not show any correlation that could possibly explain the age-related preference for geometric cues. However, unpublished data on brain imaging studies revealed impaired high-spatial frequency coding in brain regions responsible for visuospatial processing, such as the visual cortex and retrosplenial cortex, and showed gray matter atrophy in brain regions at the interface between spatial and visual coding (occipital place area and parahippocampal place area).

The ecological setup mentioned above with both a naturalistic and a virtual environment adds a completely new dimension to possible future outcome measures and also seems interesting in the context of glaucoma, since glaucoma not only appears to be restricted to retinal ganglion cell loss with subsequent visual field defects, but might also be associated with more widespread neurodegeneration [4]. 
New Developments in Optical Coherence Tomography

Peter Maloca, MD, presented his group's hardware and software innovations in optical coherence tomography (OCT) imaging. HYDRA Dual Wavelength OCT (HydraOCT) is a dual coaxial OCT system with a second light source of $1,060 \mathrm{~nm}$ in addition to the existing 870 $\mathrm{nm}$ light source. The system is based on the Spectralis OCT system (Heidelberg Engineering, Heidelberg, Germany) and can be easily integrated into clinical routine. Maloca claims that HydraOCT allows better visualization of deeper retinal structures and provides additional information, since the refractive index can also be calculated due to the two different wavelengths, thus enabling direct tissue measurements. The portable MIMO OCT device implements a very fast so-called sparse OCT scanning system and was designed for self-monitoring of patients at home $[37,38]$. The data are supposed to be analyzed with a cloud-based machine learning platform and are then displayed to patients or physicians for continuous monitoring of possible disease progression. At the same time, this would allow monitoring of large patient cohorts. The latter is applicable to both retinopathies and glaucoma.

With regard to software developments, Maloca presented their 3D speckle denoiser [39], which improves the signal-to-noise ratio not only for OCT imaging but also for 3D ultrasound, computed tomography, and possibly other imaging modalities [40-44]. This might allow a more accurate segmentation of retinal and optic nerve structures. Finally, the audience caught a glimpse of the technological potential of original 3D point cloud data being "injected" and instantly rendered into an interactive virtual reality environment [45]. The group has applied artificial intelligence (AI)-based segmentation of the data and allows the "virtual reality pilot" to interact with it. Ultimately, the virtual environment might allow the sharing of standardized mobility mazes between centers as outcome measures.

\section{Advancements in Diagnostic Imaging Modalities}

Tilman Otto from Heidelberg Engineering gave a comprehensive overview of advancements in diagnostic imaging modalities, a summary of which is clearly beyond the scope of this review. A more detailed summary is provided by della Volpe-Waizel et al. [66] in this issue of Ophthalmic Research. Basically, the imaging technologies either involve single- or two-photon interactions. Most imaging technologies are based on single-photon interactions, which include backscattering, as in conventional fundus photography, SLO, OCT, and OCT angiography imaging, and detection of fluorescence via biofluorescence or fluorescent dyes.

OCT elastography (OCE) is an imaging development that might be relevant to corneal pathologies or glaucoma. OCE allows OCT-based measurements of subtle structural fundus changes due to mechanical stress (e.g., increased intraocular pressure [IOP]) and derives information on tissue elasticity [46]. The development of the technology is at a preclinical stage. However, in the future it might shed light on pathophysiological mechanisms resulting from stress and strain on the ocular connective tissue. The latter is dependent on translaminar pressure, the anatomy of the optic nerve head, and mechanical stiffness. Regarding glaucoma trials, one might hypothesize that OCE may allow patient risk stratification for disease progression based on predisposing differences in mechanical properties of the ocular connective tissue.

\section{AI in Retinal Imaging}

Today, AI is omnipresent and ophthalmology is at its forefront. Ursula Schmidt-Erfurth outlined the tremendous development our field has experienced, starting out from conventional 2D fundus photography, having evolved into 3D OCT imaging of even subclinical pathologies over the last 25 years [47], and recently having moved into the era of deep learning since the early 2010 s. The rudiments of AI have been available since the 1950s. However, only with the increase in data volume and server capacity came a considerably improved level of operation. Vice versa, the exponential rise in available imaging data has necessitated AI to identify and quantify relevant biomarkers by providing automated segmentation, quantification of lesions, pattern recognition, prediction of recurrence or progression, and structure-function correlation, amongst other features.

Sequential neuronal networks enable a deep learning system to identify clinical features the system has not been trained with before in a so-called black box manner [48]. Even though attention maps can highlight the anatomical regions the algorithm has taken into consideration (e.g., for determining gender) [49], the clinician might still not be able to fully retrace how a neural network has come to a particular conclusion, with obvious implications for data interpretation and reliability assessment [48]. While AI has predominantly been used in retinal diseases, it is also increasingly applied to fundus photography $[50,51]$, OCT [52], and visual field evaluation [53] for glaucoma, with the ultimate goal of early diagnosis, progression analysis, and overall risk stratification, which would allow appropriate therapeutic interventions [54].
Traber et al. 


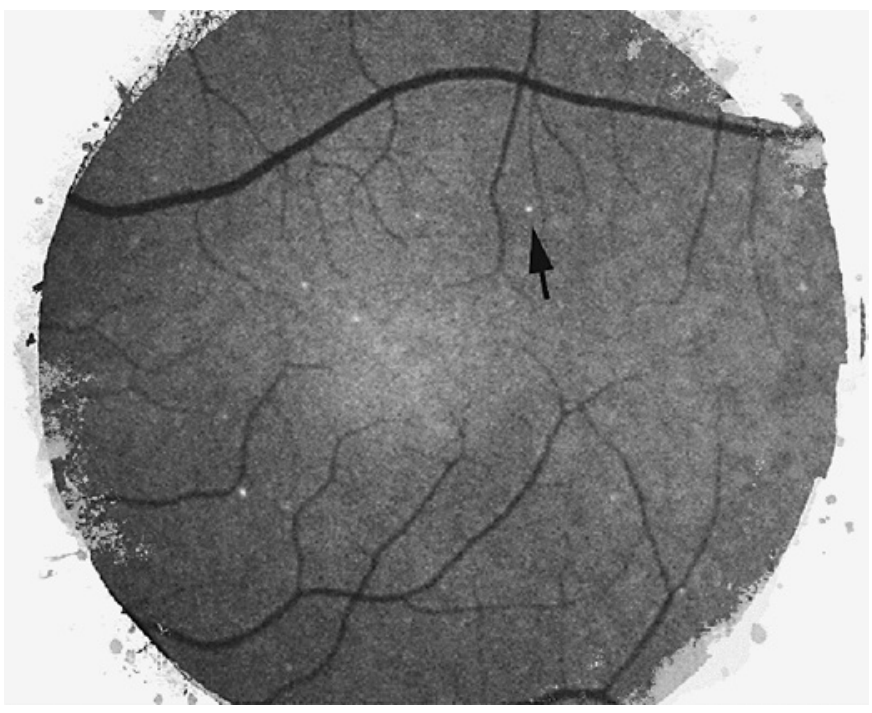

Fig. 3. Detection of apoptosing retinal cells (DARC). Scanning laser ophthalmoscopy fundus image with an ICGA setting showing hyperfluorescent DARC spots (arrow) in a glaucoma patient. Fluorescently labelled ANX776 has a high affinity to phosphatidylserine, which is exposed on the surface of cells under stress. Intravenous injection of ANX776 thus allows imaging of stressed or apoptosing retinal ganglion cells in glaucoma.

\section{Detection of Apoptosing Retinal Cells}

$M$. Francesca Cordeiro presented the evolution of the DARC (detection of apoptosing retinal cells) technology from bench to bedside. ATP-dependent "flippases" normally maintain phosphatidylserine (PS) predominantly in the cytosolic leaflet of cell membranes [55]. However, under stress or during early apoptosis, PS flips to the cell surface. The DARC technology is based on the high affinity of intravenous fluorescently labelled annexin A5 to PS, which can be detected in vivo using the ICGA settings of confocal SLO (Heidelberg Spectralis; Heidelberg Engineering, Dossenheim, Germany). Individual hyperfluorescent DARC spots (Fig. 3) are then quantified as a "DARC count" in real time.

DARC has been investigated and established in multiple rodent studies. A recently published phase I clinical trial [56] allowed proof of concept in humans. DARC proved to be a safe method for monitoring RGC apoptosis, and there was a significant difference in DARC count between healthy controls and patients with progressing glaucoma. Late diagnosis and treatment, with subsequent visual loss, represent major challenges to the management of glaucoma patients, and research is hampered by a lack of clinically meaningful endpoints and by the long

New Technologies for Outcome Measures in Glaucoma duration of clinical trials. In that context, the DARC count seems a promising early and objective clinical endpoint and might provide a new tool for testing the clinical efficacy of therapies for glaucoma and other neurodegenerative diseases [55].

\section{Induced Pluripotent Cell Technology for in vitro \\ Preclinical Testing}

Botond Roska started out from a histological drawing of the retina by Cajal, the 1906 Nobel prize winner for his milestone work on the basic principles of organization of the nervous system. Cajal described different cell morphologies, concluding that the retina is a neural network of different cell types. Today we know that these cell types have unique gene expression patterns [57-59] and that most retinal diseases are cell type specific. While the architecture of the retina is very conserved across vertebrates, the gene expression pattern is different from species to species $[57,58]$. For this reason, it appears to be difficult to develop a gene therapy in mice and translate it to humans.

Botond Roska's group instead focuses on human retinal cells. On the one hand, the group uses organ donor retinal explants to study visual processing of the retina with microchips and to build a human gene expression library of the different cell types. On the other hand, the group has managed to grow retinal organoids in a dish from skin biopsies. These retinal organoids are structurally similar to normal retinas and have turned out to show an identical rate of development to that found in published data on fetal retinal development with a gene expression map convergence around 30 weeks of gestation.

Realizing that retinal ganglion cells would undergo early apoptosis without the connecting neuron in the brain, the group has been successful in growing eye-brain organoids. GFP (green fluorescent protein) virus vector labeling of ganglion cell axons allows visualization of the organoid's optic nerve and its connections to the organoid's brain, which represents an ideal in vitro model for glaucoma research. The organoid's optic nerve can be exposed to pressure, hypoxia, or neurotrophic or neurotoxic substances to tackle neurodegeneration mechanisms in glaucoma. In addition, Botond Roska's group has not only produced 230 viral vectors targeting many cell types in the retina [60] but has also developed so-called remote control viruses. These are superinfective viruses packed on the surface of magnetic beads which, once injected into the eye, can be guided and massaged onto the retinal surface by an external magnetic field for future optimized delivery of gene therapy to the target tissue [61]. 


\section{Discussion and Conclusion}

Reduction of IOP is known to slow glaucoma progression in terms of visual field or functional loss [62-64]. Thus, IOP and standard visual fields are the endpoints accepted by the Food and Drug Administration (FDA) in studies evaluating new therapies for glaucoma [65]. However, given the fact that glaucoma is a complex disease, alternative therapeutic targets are being investigated, entailing a need for new surrogate endpoints. For its potential to detect subtle changes in the neurodegenerative activity of disease, DARC may thus be applied in trials testing neuroprotective properties of drugs.

Structural parameters in the glaucoma literature typically include retinal nerve fiber layer, ganglion cell layer, and optic disc changes. However, structural endpoints are still not established for use in clinical trials for new glaucoma drugs, as long as the structural-functional relationship at different glaucoma stages remains insufficiently characterized [65]. In the meantime, structural endpoints still may have a role in proof-ofconcept studies. In addition, it is conceivable that a combination of functional and structural endpoints will accelerate translational approaches, reduce the duration and cost of clinical trials, and thus improve visual health in the future. OCE clearly is on an exploratory level. However, the other presented refined technologies of imaging, visual function assessment, and data analysis may allow a better characterization of structure-function correlations, which is pivotal to improve study designs in the future.

The EVI special interest focus group joined forces to contribute to this long-term ambition by addressing both functional and structural measures as well as translational hot topics in glaucoma and retina research. This meeting impressively illustrated the ongoing scientific and technological progress in our field, exploring every magnitude in time and space - from picoseconds to long-term follow-up, from electrons to eye-brain organoids, and from subclinical to augmented reality. With this ever-increasing amount of information available from a single subject, it seems natural that more sophisticated, AIbased analysis and visualization of data becomes indispensable. This in turn is dependent on big data from collaborative projects, which clearly is the vision of the EVI.

\section{Acknowledgements}

The symposium thanks the EVI for support and organization, all the speakers, and the attendees at the meeting.

\section{Statement of Ethics}

The authors have no ethical conflicts to disclose.

\section{Disclosure Statement}

M. della Volpe-Waizel received consultation fees from Novartis; P. Maloca is owner of intellectual property on speckle noise analysis and the MIMO OCT discussed in the manuscript and received lecture fees from Heidelberg Engineering ${ }^{\circledR} \mathrm{GmbH}$ and Zeiss; U. Schmidt-Erfurth declares to be a consultant for Novartis, Genentech, Boehringer, and Roche; G. Rubin declares that he is a consultant for Pixium Vision SA and MeiraGTxz; M.F. Cordeiro is a named inventor on patents for DARC technology, currently owned by UCL, and is Director of Illustratum Ltd.; T. Otto declares to be Head of Technology Management - Ophthalmic Devices at Heidelberg Engineering ${ }^{\mathbb{B}}$ GmbH. R. Weleber declares the following conflicts of interest: Foundation Fighting Blindness: ViceChair for Scientific Advisory Board (SAB), Member of Executive Scientific Advisory Board (ESAB) (honorarium received); AGTC: SAB, Co-Investigator for three trials: RPE65 LCA, XLRS1, and CNGB3 ACHR; Consultant (Past): Oxford-Biomedica, Pfizer, and Novartis; Consultant (Current): NightStaRx, QLT, and 4D Molecular Therapeutics; Sanofi: Past PI and now Co-Investigator for two clinical treatment trials: USHSTAT and STARGEN; Holder of US Patent No. 8657446 Visual Field Modeling and Analysis (VFMA). A subset of his presentation material was given at a NEI/ FDA Endpoints Workshop on Age-Related Macular Degeneration and Inherited Retinal Disease held at the NEI in November 2016 [67]. L.A. Lesmes discloses financial, intellectual property, and employment interests in Adaptive Sensory Technology, Inc., which is commercializing novel devices for acuity and CS testing; A. Arleo declares to be the Head of the Essilor-ANR SilverSight Chair and to be a consultant for Essilor. H.P.N. Scholl declares the following conflicts of interest: Data Monitoring Committee: Genentech Inc./F. Hoffmann-La Roche Ltd (CHROMA and SPECTRI trials), Genzyme Corp./Sanofi, and ReNeuron Group Plc/Ora Inc.; Steering Committee: Novo Nordisk (FOCUS trial); Scientific Advisory Board: Astellas Institute for Regenerative Medicine, Gensight Biologics, Intellia Therapeutics, Inc., Ionis Pharmaceuticals, Inc., ReNeuron Group Plc/Ora Inc., Pharma Research \& Early Development (pRED) of F. Hoffmann-La Roche Ltd, and Vision Medicines, Inc.; Consultancy: Boehringer Ingelheim Pharma GmbH \& Co. KG, Daiichi Sankyo, Inc., Gerson Lehrman Group, Guidepoint, and Shire; and Co-Director of the Institute of Molecular and Clinical Ophthalmology Basel (IOB), which is constituted as a nonprofit foundation and receives funding from the University of Basel, the University Hospital Basel, Novartis, and the government of Basel-Stadt.

Except as noted above, all authors certify that they have no affiliations with or involvement in any organization or entity with any financial interest (such as honoraria; educational grants; participation in speakers' bureaus; and membership, employment, consultancies, stock ownership, or other equity interest; or any expert testimony or patent-licensing arrangements) or nonfinancial interest (such as personal or professional relationships, affiliations, knowledge, or beliefs) in the subject matter or materials discussed in this manuscript.
Traber et al. 


\section{Funding Sources}

H.P.N. Scholl was supported by an unrestricted grant from Acucela Inc., Aegerion Pharmaceuticals (Novelion Therapeutics), Kinarus AG; NightstaRx Ltd., Ophthotech Corporation, and Spark Therapeutics England, Ltd.; M. della Volpe-Waizel was supported by an unrestricted grant from the SAMW (Schweizerische Akademie der Medizinischen Wissenschaften), SNF (Swiss National Science Foundation), and Bangerter Foundation.

\section{Author Contributions}

All authors wrote the manuscript, took part in the revision process, and approved the final submission.

\section{References}

1 Tham YC, Li X, Wong TY, Quigley HA, Aung T, Cheng CY. Global prevalence of glaucoma and projections of glaucoma burden through 2040: a systematic review and meta-analysis. Ophthalmology. 2014 Nov;121(11):2081-90.

2 Weinreb RN, Aung T, Medeiros FA. The pathophysiology and treatment of glaucoma: a review. JAMA. 2014 May;311(18):1901-11.

3 Jonas JB, Aung T, Bourne RR, Bron AM, Ritch R, Panda-Jonas S. Glaucoma. Lancet. 2017 Nov;390(10108):2183-93.

4 Kasi A, Faiq MA, Chan KC. In vivo imaging of structural, metabolic and functional brain changes in glaucoma. Neural Regen Res. 2019 Mar;14(3):446-9.

5 Wey S, Amanullah S, Spaeth GL, Ustaoglu M, Rahmatnejad K, Katz LJ. Is primary open-angle glaucoma an ocular manifestation of systemic disease? Graefes Arch Clin Exp Ophthalmol. 2019 Apr;257(4):665-73.

6 Varma R, Lee PP, Goldberg I, Kotak S. An assessment of the health and economic burdens of glaucoma. Am J Ophthalmol. 2011 Oct; 152(4):515-22.

7 Lesmes LA, Lu ZL, Baek J, Albright TD. Bayesian adaptive estimation of the contrast sensitivity function: the quick CSF method. J Vis. 2010 Mar; 10(3):17.1-21

8 Lin S, Mihailovic A, West SK, Johnson CA, Friedman DS, Kong X, et al. Predicting Visual Disability in Glaucoma with Combinations of Vision Measures. Transl Vis Sci Technol. 2018 Apr;7(2):22.

9 Hot A, Dul MW, Swanson WH. Development and evaluation of a contrast sensitivity perimetry test for patients with glaucoma. Invest Ophthalmol Vis Sci. 2008 Jul;49(7):3049-57.

10 Ross JE, Bron AJ, Clarke DD. Contrast sensitivity and visual disability in chronic simple glaucoma. Br J Ophthalmol. 1984 Nov;68(11): 821-7.

11 Stamper RL. The effect of glaucoma on central visual function. Trans Am Ophthalmol Soc. 1984;82:792-826.

12 Nguyen AM, Mihailovic A, Friedman DS, Ramulu PY. Comparison of contrast sensitivity, visual acuity, and the contrast sensitivity function as predictors of gait in glaucoma. Invest Ophthalmol Vis Sci. 2016;57(12):1953.

13 Hou F, Lesmes L, Bex P, Dorr M, Lu ZL. Using 10AFC to further improve the efficiency of the quick CSF method. J Vis. 2015;15(9):2.
14 Lesmes L, Jackson M, Bex P. Visual function endpoints to enable dry AMD clinical trials. Drug Discov Today Ther Strateg. 2013; 10(1):e43-50.

15 Bach M. The Freiburg Visual Acuity test - automatic measurement of visual acuity. Optom Vis Sci. 1996 Jan;73(1):49-53.

16 Lesmes LA, Dorr M. Active learning for visual acuity testing. Proceedings of the 2 nd International Conference on Applications of Intelligent Systems - APPIS '19. Las Palmas de Gran Canaria, Spain: ACM Press; 2019; pp. $1-6$.

17 Crossland MD, Culham LE, Kabanarou SA, Rubin GS. Preferred retinal locus development in patients with macular disease. Ophthalmology. 2005 Sep;112(9):1579-85.

18 Bainbridge JW, Smith AJ, Barker SS, Robbie S, Henderson R, Balaggan K, et al. Effect of gene therapy on visual function in Leber's congenital amaurosis. N Engl J Med. 2008 May;358(21):2231-9.

19 Bainbridge JW, Mehat MS, Sundaram V, Robbie SJ, Barker SE, Ripamonti C, et al. Long-term effect of gene therapy on Leber's congenital amaurosis. N Engl J Med. 2015 May;372(20):1887-97.

20 Lima VC, Prata TS, De Moraes CG, Kim J, Seiple W, Rosen RB, et al. A comparison between microperimetry and standard achromatic perimetry of the central visual field in eyes with glaucomatous paracentral visual-field defects. Br J Ophthalmol. 2010 Jan;94(1):64-7.

21 Oztürk F, Yavas GF, Küsbeci T, Ermis SS. A comparison among Humphrey field analyzer, microperimetry, and Heidelberg Retina Tomograph in the evaluation of macula in primary open angle glaucoma. J Glaucoma. 2008 Mar;17(2):118-21.

22 Orzalesi N, Miglior S, Lonati C, Rosetti L. Microperimetry of localized retinal nerve fiber layer defects. Vision Res. 1998 Mar;38(5): $763-71$.

23 Matsuura M, Murata H, Fujino Y, Hirasawa K, Yanagisawa M, Asaoka R. Evaluating the Usefulness of MP-3 Microperimetry in Glaucoma Patients. Am J Ophthalmol. 2018 Mar; 187:1-9.

24 Rao HL, Januwada M, Hussain RS, Pillutla LN, Begum VU, Chaitanya A, et al. Comparing the Structure-Function Relationship at the Macula with Standard Automated Perim- etry and Microperimetry. Invest Ophthalmol Vis Sci. 2015 Dec;56(13):8063-8.

25 Rao HL, Hussain RS, Januwada M, Pillutla LN, Begum VU, Chaitanya A, et al. Structural and functional assessment of macula to diagnose glaucoma. Eye (Lond). 2017 Apr;31(4): 593-600.

26 Sato S, Hirooka K, Baba T, Tenkumo K, Nitta E, Shiraga F. Correlation between the ganglion cell-inner plexiform layer thickness measured with cirrus HD-OCT and macular visual field sensitivity measured with microperimetry. Invest Ophthalmol Vis Sci. $2013 \mathrm{Apr}$; 54(4):3046-51

27 Klamann MK, Grünert A, Maier AK, Gonnermann J, Joussen AM, Huber KK. Comparison of functional and morphological diagnostics in glaucoma patients and healthy subjects. Ophthalmic Res. 2013;49(4):192-8.

28 Ratnarajan G, Jolly JK, Yusuf IH, Salmon JF. The effect of trabeculectomy surgery on the central visual field in patients with glaucoma using microperimetry and optical coherence tomography. Eye (Lond). 2018 Aug;32(8): 1365-71.

29 Okada K, Watanabe W, Koike I, Tsumamoto Y, Mishima HK. Alternative method of evaluating visual field deterioration in very advanced glaucomatous eye by microperimetry. Jpn J Ophthalmol. 2003 Mar-Apr;47(2):17881.

30 Longhin E, Convento E, Pilotto E, Bonin G, Vujosevic S, Kotsafti O, et al. Static and dynamic retinal fixation stability in microperimetry. Can J Ophthalmol. 2013 Oct;48(5): 375-80.

31 Shi Y, Liu M, Wang X, Zhang C, Huang P. Fixation behavior in primary open angle glaucoma at early and moderate stage assessed by the MicroPerimeter MP-1. J Glaucoma. 2013 Feb;22(2):169-73.

32 Weleber RG, Smith TB, Peters D, Chegarnov EN, Gillespie SP, Francis PJ, et al. VFMA: Topographic Analysis of Sensitivity Data from Full-Field Static Perimetry. Transl Vis Sci Technol. 2015 Apr;4(2):14.

33 Weleber RG, Pennesi ME, Wilson DJ, Kaushal S, Erker LR, Jensen L, et al. Results at 2 Years after Gene Therapy for RPE65-Deficient Leber Congenital Amaurosis and Severe EarlyChildhood-Onset Retinal Dystrophy. Ophthalmology. 2016 Jul;123(7):1606-20. 
34 Lester AW, Moffat SD, Wiener JM, Barnes CA, Wolbers T. The Aging Navigational System. Neuron. 2017 Aug;95(5):1019-35.

35 Bécu M, Sheynikhovich D, Tatur G, Agathos CP, Bologna LL, Sahel JA, et al. Age-related preference for geometric spatial cues during real-world navigation. Nat Hum Behav. 2019 [Epub ahead of print].

36 Bécu M. Impact of healthy aging on spatial cognition. Spatial navigation and gaze dynamics in ecological conditions [ $\mathrm{PhD}$ thesis]. Paris: Sorbonne University; 2018.

37 Maloca P, Hasler PW, Barthelmes D, Arnold P, Matthias M, Scholl HP, et al. Safety and Feasibility of a Novel Sparse Optical Coherence Tomography Device for Patient-Delivered Retina Home Monitoring. Transl Vis Sci Technol. 2018 Jul;7(4):8.

38 Quellec G, Kowal J, Hasler PW, Scholl HP, Zweifel S, Konstantinos B, et al. Feasibility of support vector machine learning in age-related macular degeneration using small sample yielding sparse optical coherence tomography data. Acta Ophthalmol. 2019 Aug; 97(5):e719-28.

39 Gyger C, Cattin R, Hasler PW, Maloca P. Three-dimensional speckle reduction in optical coherence tomography through structural guided filtering. Opt Eng. 2014 Jul;53(7): 073105.

40 Maloca P, Gyger C, Hasler PW. A pilot study to image the vascular network of small melanocytic choroidal tumors with speckle noisefree 1,050-nm swept source optical coherence tomography (OCT choroidal angiography). Graefes Arch Clin Exp Ophthalmol. 2016 Jun; 254(6):1201-10.

41 Maloca P, Gyger C, Hasler PW. A pilot study to compartmentalize small melanocytic choroidal tumors and choroidal vessels with speckle-noise free 1,050 nm swept source optical coherence tomography (OCT choroidal "tumoropsy"). Graefes Arch Clin Exp Ophthalmol. 2016 Jun;254(6):1211-9.

42 Maloca P, Gyger C, Schoetzau A, Hasler PW. Ultra-Short-Term Reproducibility of Speckle-Noise Freed Fluid and Tissue Compartmentalization of the Choroid Analyzed by Standard OCT. Transl Vis Sci Technol. 2015 Nov;4(6):3.

43 Maloca PM, Spaide RF, Rothenbuehler S, Scholl HP, Heeren T, Ramos de Carvalho JE, et al. Enhanced resolution and speckle-free three-dimensional printing of macular optical coherence tomography angiography. Acta Ophthalmol. 2019 Mar;97(2):e317-9.

44 Rothenbuehler SP, Maloca P, Scholl HP, Gyger C, Schoetzau A, Kuske L, et al. Threedimensional Analysis of Submacular Perforating Scleral Vessels by Enhanced Depth Imaging Optical Coherence Tomography. Retina. 2018 Jun;38(6):1231-7.
45 Maloca PM, de Carvalho JE, Heeren T, Hasler PW, Mushtaq F, Mon-Williams M, et al. High-Performance Virtual Reality Volume Rendering of Original Optical Coherence Tomography Point-Cloud Data Enhanced with Real-Time Ray Casting. Transl Vis Sci Technol. 2018 Jul;7(4):2.

46 Fazio MA, Clark ME, Bruno L, Girkin CA. In vivo optic nerve head mechanical response to intraocular and cerebrospinal fluid pressure: imaging protocol and quantification method. Sci Rep. 2018 Aug;8(1):12639.

47 Fujimoto J, Swanson E. The Development, Commercialization, and Impact of Optical Coherence Tomography. Invest Ophthalmol Vis Sci. 2016 Jul;57(9):OCT1-13.

48 Schmidt-Erfurth U, Sadeghipour A, Gerendas BS, Waldstein SM, Bogunović H. Artificial intelligence in retina. Prog Retin Eye Res. 2018 Nov;67:1-29.

49 Poplin R, Varadarajan AV, Blumer K, Liu Y, McConnell MV, Corrado GS, et al. Prediction of cardiovascular risk factors from retinal fundus photographs via deep learning. Nat Biomed Eng. 2018 Mar;2(3):158-64.

50 Ting DS, Tan GS, Agrawal R, Yanagi Y, Sie NM, Wong CW, et al. Optical Coherence Tomographic Angiography in Type 2 Diabetes and Diabetic Retinopathy. JAMA Ophthalmol. 2017 Apr;135(4):306-12.

51 Li Z, He Y, Keel S, Meng W, Chang RT, He M. Efficacy of a Deep Learning System for Detecting Glaucomatous Optic Neuropathy Based on Color Fundus Photographs. Ophthalmology. 2018 Aug;125(8):1199-206

52 Christopher M, Belghith A, Weinreb RN, Bowd C, Goldbaum MH, Saunders LJ, et al. Retinal Nerve Fiber Layer Features Identified by Unsupervised Machine Learning on Optical Coherence Tomography Scans Predict Glaucoma Progression. Invest Ophthalmol Vis Sci. 2018 Jun;59(7):2748-56.

53 Wang M, Pasquale LR, Shen LQ, Boland MV, Wellik SR, De Moraes CG, et al. Reversal of Glaucoma Hemifield Test Results and Visual Field Features in Glaucoma. Ophthalmology. 2018 Mar;125(3):352-60

54 Ting DS, Pasquale LR, Peng L, Campbell JP, Lee AY, Raman R, et al. Artificial intelligence and deep learning in ophthalmology. Br J Ophthalmol. 2019 Feb;103(2):167-75.

55 Yap TE, Donna P, Almonte MT, Cordeiro MF. Real-Time Imaging of Retinal Ganglion Cell Apoptosis. Cells. 2018 Jun;7(6):E60.

56 Cordeiro MF, Normando EM, Cardoso MJ, Miodragovic S, Jeylani S, Davis BM, et al. Real-time imaging of single neuronal cell apoptosis in patients with glaucoma. Brain. 2017 Jun;140(6):1757-67.
57 Macosko EZ, Basu A, Satija R, Nemesh J, Shekhar K, Goldman M, et al. Highly Parallel Genome-Wide Expression Profiling of Individual Cells Using Nanoliter Droplets. Cell. 2015 May;161(5): 1202-14.

58 Peng YR, Shekhar K, Yan W, Herrmann D, Sappington A, Bryman GS, et al. Molecular Classification and Comparative Taxonomics of Foveal and Peripheral Cells in Primate Retina. Cell. 2019 Feb;176(5):1222-1237.e22.

59 Siegert S, Cabuy E, Scherf BG, Kohler H, Panda S, Le YZ, et al. Transcriptional code and disease map for adult retinal cell types. Nat Neurosci. 2012 Jan;15(3):487-95, S1-2.

60 Jüttner J, Szabo A, Gross-Scherf B, Morikawa RK, Rompani SB, Teixeira M, et al. Targeting neuronal and glial cell types with synthetic promoter AAVs in mice, non-human primates, and humans. bioRxiv. 2018;(Jan): 434720.

61 Schubert R, Trenholm S, Balint K, Kosche G, Cowan CS, Mohr MA, et al. Virus stamping for targeted single-cell infection in vitro and in vivo. Nat Biotechnol. 2018 Jan;36(1):81-8.

62 Wickström K, Moseley J. Biomarkers and Surrogate Endpoints in Drug Development: A European Regulatory View. Invest Ophthalmol Vis Sci. 2017 May;58(6):BIO27-33.

63 Heijl A, Leske MC, Bengtsson B, Hyman L, Bengtsson B, Hussein M; Early Manifest Glaucoma Trial Group. Reduction of intraocular pressure and glaucoma progression: results from the Early Manifest Glaucoma Trial. Arch Ophthalmol. 2002 Oct;120(10): 1268-79.

64 Garway-Heath DF, Crabb DP, Bunce C, Lascaratos G, Amalfitano F, Anand N, et al. Latanoprost for open-angle glaucoma (UKGTS): a randomised, multicentre, placebocontrolled trial. Lancet. 2015 Apr;385(9975): 1295-304.

65 Weinreb RN, Kaufman PL. Glaucoma research community and FDA look to the future, II: NEI/FDA Glaucoma Clinical Trial Design and Endpoints Symposium: measures of structural change and visual function. Invest Ophthalmol Vis Sci. 2011 Oct;52(11): 7842-51.

66 Della Volpe-Waizel M, Traber GL, Maloca P, Zinkernagel M, Schmidt-Erfurth U, Rubin G, et al. New Technologies for Outcome Measures in Retinal Disease: Review from the European Vision Institute Special Interest Focus Group. Ophthalmic Res. 2019 [Epub ahead of print].

67 Csaky K, Ferris F 3rd, Chew EY, Nair P, Cheetham JK, Duncan JL. Report from the NEI/FDA Endpoints Workshop on Age-Related Macular Degeneration and Inherited Retinal Diseases. Invest Ophthalmol Vis Sci. 2017 Jul;58(9):3456-63. 